\title{
A modified new Homotopy Perturbation Method for solving linear integral equations - differential
}

\author{
Aisan Khojasteh ${ }^{1, ~}$, Mahmoud Paripour ${ }^{2}$ \\ ${ }^{1}$ M. A. Applied Mathematics, Science and Research Branch, Islamic Azad University, Broujerd, Iran \\ ${ }^{2}$ Assistant Professor of Applied Mathematics, Hamedan University of Technology, Hamedan, Iran \\ Email address: \\ aisan.khojasteh@yahoo.com (A. Khojasteh),Mahmoud_paripour@yahoo.com (M. Paripour)
}

To cite this article:

Aisan Khojasteh, Mahmoud Paripour. A Modified New Homotopy Perturbation Method for Solving Linear Integral Equations Differential. American Journal of Applied Mathematics. Vol. 2, No. 3, 2014, pp. 79-84. doi: 10.11648/j.ajam.20140203.12

\begin{abstract}
Mathematical modeling of real-life problems usually results in functional equations, such as ordinary or partial differential equations, integral and integral-differential equations etc. The theory of integral equation is one of the major topics of applied mathematics. In this paper a new Homotopy Perturbation Method (HPM) is introduced to obtain exact solutions of the systems of integral equations-differential and is provided examples for the accuracy of this method. This paper presents an introduction to new method of HPM, then introduces the system of integral - differential linear equations and also introduces applications and literature. In second section we will introduce categorizations of averaging integral differential and several methods to solve this kind of achievement. The third section introduces a new method of HPM. Fourth section determines quarter of integral - differential equations by using HPM. Therefore, we provide Conclusion and some examples that illustrate the effectiveness and convenience of the proposed method.
\end{abstract}

Keywords: New Homotopy Perturbation Method, Systems of Integral Equations - Differential

\section{Introduction}

The homotopy analysis method (HAM) was proposed by Liao. In this method, the solution is considered as the summation of an infinite series, which usually converges rapidly to the exact solution. The HAM is based on homotopy, a fundamental concept in topology and differential geometry. Briefly speaking, by means of the HAM, one constructs a continuous mapping of an initial guess approximation to the exact solution of considered equations. An auxiliary linear operator is chosen to construct such kind of continuous mapping, and an auxiliary parameter is used to ensure the convergence of solution series. The method enjoys great freedom in choosing initial approximations and auxiliary linear operators. The approximations obtained by the HAM are uniformly valid not only for small parameters, but also for very large parameters. Until recently, the application of the homotopy analysis method in nonlinear problems has been devoted by scientists and engineers.

\section{Systems of Differential Equations}

Newton's second law of motion for point particles is one of the first deferential equations to ever be written. Even this early example of a deferential equation consists not of a single equation but of a system of three equations on three unknowns. The unknown functions are the particle three coordinates in space as function of time. One important difficulty to solve a deferential system is that the equations in a system are usually coupled. One cannot solve for one unknown function without knowing the other unknowns. In this Chapter we study how to solve the system in the particular case that the equations can be uncoupled. We call such systems diagonalizable. Explicit formulas for the solutions can be written in this case. Later we generalize this idea to systems that cannot be uncoupled.

\subsection{Linear Differential Systems}

We introduce a linear deferential system with variable coefficients. We present an initial value problem for such systems and we state that initial value problems always have a unique solution. The proof is based on a generalization of the Picard-Lindel of iteration used in Section 1.6. We then introduce the concepts of fundamental solution, general solution, fundamental matrix, and Wronskian of solutions to a linear system. 


\section{Nonlinear Differential and Integral Equations}

Within recent years interest in nonlinear equations has grown enormously. They are extremely important as basic equations in many areas of mathematical physics, and they have received renewed attention because of progress in their solution by machines. This volume undertakes a definition of the field, indicating advances that have been made up through 1960. The author's position is that while the advent of machines has resulted in much new knowledge, one should not disregard analytical methods, since the solution of nonlinear equations possesses singularities which only the analytical method (as based upon the work of Poincare, Liapounoff, Pain eve and Goursatl can discover.

After a general survey of the problem presented by nonlinear equations, the author discusses the differential equation of the first order, following this by chapters on the Riccati equation (as a bridge between linear and nonlinear equations) and existence theorems, with special reference to Cauchy's method. Second order equations are introduced via Volterra's problem and the problem of pursuit, and succeeding chapters cover elliptic integrals and functions and theta functions; differential equations of the second order; and second order differential equations of the polynomial class, with special reference to Painleve transcendent. The technique of continuous analytical continuation is shown, while phenomena of the phase plane are studied as an introduction to nonlinear mechanics. Nonlinear 111echanics is then discussed, with various classical equations like Van der Pol's equations, Emden's equation, and the Duffing problem. The remaining chapters are concerned with nonlinear integral equations, problems from the calculus of variations, and numerical integration of nonlinear equations. Throughout the book the results of distinguished analysis of the past and modern machine computations are both taken into account. Despite the thoroughness of its coverage this is a very fine introduction to this important area of mathematics, and it can easily be followed by the mathematically sophisticated reader who knows very little about nonlinear equations for example:

$$
\begin{gathered}
x(t)=\int_{a}^{b} k(t, s) x^{(n)} d s \\
x^{(i)}(0)=d_{i} \quad 0 \leq i \leq n
\end{gathered}
$$

\section{Method for Ordinary Differential Equations}

This part will introduce the reader to the terminology and notation of differential equations. Students will also be reminded of some of the elementary solution methods they are assumed to have encountered in an undergraduate course on the subject. At the conclusion of this review one should have an idea of what it means to 'solve' a differential equation and some confidence that they could construct a solution to some simple and special types of differential equations for example:

$$
\begin{gathered}
x^{(n)}(t)=y(t)+\int_{a}^{s} k(t, s) x(s) d s \\
x^{(i)}(0)=d_{i} \quad 0 \leq i \leq n
\end{gathered}
$$

At the core of each of $K(t, s),(3-1),(2-1)$ the equations, the equations say that each and every one square homepage. Obviously, each of the above equations, the kernel and the function $\mathrm{y}(\mathrm{t})$ are known such as:

1) $\quad\left(K^{*}\right)^{*}=k$

2) $\left(\lambda K^{*}\right)=\lambda k^{*}$

3) $(K h)^{*}=h^{*} k^{*}$

4) $\left(K^{*} x, y\right)^{*}=\left(x, k^{*} y\right)$

In case kernel $\mathrm{h}$ and $\mathrm{k}$ is continuous, then:

$(K L)^{*}(s, t)=\overline{(k l)(t, s)}$

$$
\begin{aligned}
& =\int_{a}^{b} \overline{k(t, u) L(u, s)} d u \\
& =\int_{a}^{b} \overline{k(t, u) L(u, s)} d u \\
& =\int_{a}^{b} L^{*}(s, u) K^{*}(u, t) d u \\
& =L^{*} K^{*}(s, t)
\end{aligned}
$$

Therefore, we consider the following differential general form of an equation integral:

$$
\begin{gathered}
P(t) y^{\prime \prime}(t)+Q(t) y^{\prime}(t)+R(t) y(t)+\lambda \int_{a}^{b} k(t, s) y(s) d s=g(t) \\
C y(r)+D y^{\prime}(r) \\
r=\left(r_{1}, r_{2}, \ldots, r_{m}\right)^{T} \quad a \leq r_{i} \leq b, \quad 1 \leq i \leq m \\
y(r)=\left(y(r)_{1}, \ldots, y\left(r_{m}\right)^{T}\right. \\
y^{\prime}(r)=\left(y^{\prime}(r)_{1}, \ldots, y^{\prime}\left(r_{m}\right)^{T}\right.
\end{gathered}
$$




\section{The Concept of the Integral Equation}

Integral-differential equations appear in many scientific applications, especially when we convert initial value problems or boundary value problems to integral equations. The integral-differential equations contain both integral and differential operators. The derivatives of the unknown functions may appear to any order. In classifying integral-differential equations, we will follow the same category used before. Integral equations occur in a variety of applications, often being obtained from a differential equation. The reason for doing this is that it may make solution of the problem easier or, sometimes, enable us to prove fundamental results on the existence and uniqueness of the solution.

\section{Fredholm Integral Equations}

For Fredholm integral equations, the limits of integration are fixed. Moreover, the unknown function $u(x)$ may appear only inside integral equation.

$$
\begin{gathered}
P(t) y^{\prime \prime}(t)+Q(t) y^{\prime}(t)+R(t) y(t)+\lambda \int_{a}^{b} k(t, s) y(s) d s=g(t) \\
C y(r)+D y^{\prime}(r) \\
r=\left(r_{1}, r_{2}, \ldots, r_{m}\right)^{T} \quad a \leq r_{i} \leq b \quad 1 \leq i \leq m \\
y(r)=\left(y(r)_{1}, \ldots, y\left(r_{m}\right)^{T}\right. \\
y^{\prime}(r)=\left(y^{\prime}(r)_{1}, \ldots, y^{\prime}\left(r_{m}\right)^{T}\right.
\end{gathered}
$$

If you have equation (2.1) we consider the following assumptions:

$P(t)=1, \quad Q(t)=R(t)=0, a=0, \quad b=1, C=1, \quad D=1$,

$r=(0,1)^{T}, \quad e=(0,0)^{T}$

We have the following equation:

$$
\begin{gathered}
y^{\prime \prime}(t)+\lambda \int_{0}^{1} k(t, s) y(s) d s=g(t) \\
y(0)=y(1)=0
\end{gathered}
$$

According to the similarity of the methods for solving problems of linear ordinary differential equations, a finite difference approximation to the derivatives to replace solutions, as well as replacing the integral with a base of numerical integration with appropriate accuracy.

By introducing $f(t, y(t))=g(t)-\lambda \int_{0}^{1} k(t, s) y(s) d s \quad$ the equation 3 can be written as follows. $y^{\prime \prime}(t)=f(t, y(t)) \quad y(0)=y(1)=0$

If the two sides of equation (2.3) in the interval of $[0, t]$ integration, we will have:

$$
y^{\prime}(t)=y_{1}+\int_{0}^{t} f(s, y(s)) d s
$$

Therefore, with replacement u t, we have:

$$
y^{\prime}(u)=y_{1}+\int_{0}^{u} f(s, y(s)) d s
$$

integrating of gates re $[0, t]$ we have:

$$
\int_{0}^{t} y^{\prime}(u) d u=\int_{0}^{t} y_{1} d u+\int_{0}^{t} \int_{0}^{u} f(s, y(s)) d s d u
$$

In which case the following relation will be achieved:

$$
y(t)=y_{0}+y_{1} t+\int_{0}^{t} \int_{0}^{u} f(s, y(s)) d s d u
$$

Considering this $0 \leq \mathrm{s} \leq \mathrm{u} \leq$ we have:

$$
\begin{gathered}
y(t)=A+B t+\int_{0}^{t} f(s, y(s)) d s \int_{s}^{t} d u \\
=A+B t+\int_{0}^{t}(t-s) f(s, y(s)) d s
\end{gathered}
$$

Therefore, $B=-\int_{0}^{1}(1-s) f(s, y(s)) d s$

We have with replacement values of A and B in (2.6)

$$
y(t)=-t \int_{0}^{1}(1-s) f(s, y(s)) d s+\int_{0}^{t}(t-s) f(s, y(s)) d s
$$

For example:

$$
\begin{gathered}
G(t, s)= \begin{cases}-s(1-t) & o \leq S \leq t \\
-t(1-s) & t \leq S \leq 1\end{cases} \\
y(t)=-t \int_{0}^{1}(1-s)\left(g(s)-\lambda \int_{0}^{1} k(s, u) y(u) d u\right) d s \\
+\int_{0}^{t}(t-s)\left(g(s)-\lambda \int_{0}^{1} k(s, u) y(u) d u\right) d s \\
=\int_{0}^{1}-t(1-s) g(s) d s
\end{gathered}
$$




$$
\begin{gathered}
+\lambda \int_{0}^{1} \int_{0}^{1} t(1-s) k(s, u) y(u) d u d s \\
+\int_{0}^{t}(t-s) g(s) d s-\lambda \int_{0}^{t} \int_{0}^{1}(t-s) k(s, u) y(u) d u d s \\
=\int_{0}^{t}-t(1-s) g(s) d s+\int_{0}^{1}-t(1-s) g(s) d s \\
+\lambda \int_{0}^{t} \int_{0}^{1} t(1-s) k(s, u) y(u) d u d s+\lambda \int_{t}^{1} \int_{0}^{1} t(1-s) k(s, u) y(u) d u d s \\
+\int_{0}^{t}(t-s) g(s) d s-\lambda \int_{0}^{t} \int_{0}^{1}(t-s) k(s, u) y(u) d u d s \\
\int_{0}^{t}-t(1-s) g(s) d s+\int_{0}^{t}(t-s) g(s) d s=\int_{0}^{t}-s(1-t) g(s) d s \\
=\int_{0}^{t} G(t, s) g(s) d s
\end{gathered}
$$

also:

$$
\begin{gathered}
\int_{0}^{t} G(t, s) g(s) d s+\int_{t}^{1}-t(1-s)(g(s) d s \\
=\int_{0}^{1} G(t, s) g(s) d s
\end{gathered}
$$

We have with replacement in:

$$
y(t)=\int_{0}^{1} G(t, s) g(s) d s
$$

$$
\begin{aligned}
& +\lambda \int_{0}^{t} \int_{0}^{1} t(1-s) k(s, u) y(u) d u d s+\lambda \int_{t}^{1} \int_{0}^{1} t(1-s) k(s, u) y(u) d u d s \\
& +\lambda \int_{0}^{t} \int_{0}^{1}(s-t) k(s, u) y(u) d u d s
\end{aligned}
$$

$$
\begin{gathered}
\lambda \int_{0}^{t} \int_{0}^{1} t(1-s) k(s, u) y(u) d u d s+\lambda \int_{0}^{t} \int_{0}^{1}(s-t) k(s, u) y(u) d u d s \\
=\lambda \int_{0}^{t} \int_{0}^{1} s(1-t) k(s, u) y(u) d u d s
\end{gathered}
$$

$$
=-\lambda \int_{0}^{t} \int_{0}^{1} G(t, s) k(s, u) y(u) d u d s
$$

We have with replacement in (14)

$$
\begin{gathered}
y(t)=\int_{0}^{1} G(t, s) g(s) d s-\lambda \int_{0}^{t} \int_{0}^{1} G(t, s) k(s, u) y(u) d u d s \\
-\lambda \int_{t}^{1} \int_{0}^{1}-t(1-s) k(s, u) y(u) d u d s
\end{gathered}
$$

Or

$$
y(t)=\int_{0}^{1} G(t, s) g(s) d s-\lambda \int_{0}^{1} \int_{0}^{1} G(t, s) k(s, u) y(u) d u d s
$$

Therefore,

$$
y(t)+\lambda \int_{0}^{1} \int_{0}^{1} G(t, s) k(s, u) y(u) d u d s=\int_{0}^{1} G(t, s) g(s) d s
$$

We write the Fredholm Integral Equations

$$
\begin{gathered}
y(t)+\lambda \int_{0}^{1} H(t, u) y(u) d u=V(t) \\
H(t, u)=\int_{0}^{1} G(t, s) k(s, u) d s \\
V(t)=\int_{0}^{1} G(t, s) g(s) d s
\end{gathered}
$$

\section{Conclusion}

In this paper, the divided differences method is applied to solve the linear Fredholm integral equation of the second kind. In this method, the cosecants of divided differences are given by solving a system of equations. In the comparison, the proposed method is better than the Adomian's decomposition method to approximate the exact solution. The advantage of the proposed method over other methods is that the integral equation is solved by having support points of the solution of integral equation. The proposed method is a powerful procedure for solving linear integral equations. The examples analyzed illustrate the ability and reliability of the method presented in this paper and reveals that this one is very simple and effective. The obtained solutions, in comparison with exact solutions admit a remarkable accuracy. Results indicate that the convergence rate is very fast, and lower approximations can achieve high accuracy 
Example:

$$
\begin{gathered}
\frac{d x_{1}(t)}{d t}=t^{4}-t^{3}-2 t^{2}-6+\left(3 t^{2}-6 t+7\right) x_{1}(t) 2 t^{2}(t+1) x_{2}(t) \\
\quad+\int_{0}^{t}\left(\left(S^{3}-t^{3}\right) x_{1}(s)+t^{2}\left(s^{2}-t^{2}\right) x_{2}(s)\right) \quad, x_{1}(0)=1 \\
\frac{d x_{2}(t)}{d t}=-t^{4}-3 t^{3}+2+2(t-1) x_{1}(t)+\left(2 t^{4}+2 t^{3}+2 t^{2}-1\right) x_{2}(t) \\
+\int_{0}^{t}\left(\left(S^{2}-t^{2}\right) x_{1}(s)+t^{2}\left(s^{2}+t^{2}\right) x_{2}(s)\right) d s, x_{2}(0)=1
\end{gathered}
$$

For solve the system with new Homotopy deviation, Homotpy the following build:

$$
\begin{gathered}
\frac{d x_{1}(t)}{d t}=x_{1,0}(t)-p\left(x_{1,0}(t)-t^{4}+t^{3}+2 t^{2}+6-\left(3 t^{2}-6 t+7\right) x_{1}(t)\right. \\
\left.-2 t^{2}(t+1) x_{2}(t)-\int_{0}^{t}\left(\left(S^{3}-t^{3}\right) x_{1}(s)+t^{2}\left(s^{2}-t^{2}\right) x_{2}(s)\right) d s\right) \\
\frac{d x_{2}(t)}{d t}=x_{2,0}(t)-p\left(x_{2,0}(t)+t^{4}+3 t^{2}-2-2(t-1) x_{1}(t)\right. \\
\left.-\left(2 t^{4}+2 t^{3}+2 t^{2}-1\right) x_{2}(t)-\int_{0}^{t}\left(\left(S^{2}-t^{2}\right) x_{1}(s)+t^{2}\left(s^{2}+t^{2}\right) x_{2}(s)\right) d s\right)
\end{gathered}
$$

For example:

$$
\begin{gathered}
X_{1,0}(t)=\sum_{n=0}^{\infty} \alpha_{n} p_{n}(t) \\
X_{2,0}(t)=\sum_{n=0}^{\infty} \beta_{n} P_{n}(t) \\
P_{i}(t)=t^{i}, X_{1}(0)=X_{2}(0)=1
\end{gathered}
$$

In fact, we have:

$$
\begin{gathered}
X_{1}(t)=1+\sum_{n=0}^{\infty} \frac{\alpha_{n}}{n+1} t^{n+1}-P\left(\sum_{n=0}^{\infty} \frac{\alpha_{n}}{n+1} t^{n+1}-\frac{t^{5}}{5}+\frac{t^{4}}{4}+\frac{2 t^{3}}{3}\right. \\
\left.\left.X_{1}(\tau)+\tau^{2}\left(s^{2}-\tau^{2}\right) X_{2}(\tau)\right) d s d \tau\right), \\
-\int_{0}^{t}\left(2(s-1) X_{1}(s)+\left(2 S^{4}+2 S^{3}+2 S^{2}-1\right) X_{2}(s)\right) d s \\
X_{2}(t)=1+\sum_{n=0}^{\infty} \frac{\beta_{n}}{n+1} t^{n+1}-P\left(\sum_{n=0}^{\infty} \frac{\beta_{n}}{n+1} t^{n+1}+\frac{t^{5}}{5} t^{3}-2 t\right. \\
\left.-\int_{0}^{t} \int_{0}^{\tau}\left(\left(S^{2}-\tau^{2}\right) X_{1}(\tau)+\tau^{2}\left(S^{2}+\tau^{2}\right) X_{2}(\tau)\right) d s d \tau\right) .
\end{gathered}
$$

\section{References}

[1] Abbas bandy, S., 2006. Numerical solutions of the integral equations: homotopy perturbation method and Adomain's decomposition method. Applied Mathematics and Computation 173, 493-500.

[2] Belendez, A., Belendez, T., Marquez, A., Neipp, C., 2008. Application of He's homotopy perturbation method to conservative truly nonlinear oscillators. Chaos, Solitions and Fractals 37 (3), 770-780.

[3] Biazar, J., Ghazvini, H., 2007. Exact solutions for nonlinear Schrodinger equations by He's homotopy perturbation method. Physics Letter A 366, 79-84.

[4] Biazar, J., Ghazvini, H., 2008. Numerical solutions for special nonlinear Fredholm integral equation by HPM. Applied Mathematics and Computation 195, 681-687.

[5] Biazar, J., Ghazvini, H., 2009. He's homotopy perturbation method for solving system of Volterra integral equations of the second Kind. Chaos, Solitons and Fractals 39, 770-777.

[6] Bo, T.L., Xie, L., Zheng, X.J., 2007. Numerical approach to wind ripple in desert International Journal of Nonlinear Sciences and Numerical Simulation 8(2), 223-228.

[7] Ganji, D.D., 2006. The application of He's homotopy perturbation method to nonlinear equations arising in heat transfer. Physics Letter A 355, 337-341.

[8] Ganji, D.D., 2006. The application of He's homotopy perturbation and variational iteration methods to nonlinear heat transfer and porous media equations. Journal of Computational and Applied Mathematics 207, 24-34.

[9] Golbabai, A., Javidi, M., 2007. Application of He's homotopy perturbation method for solving eighth-order boundary value problems. Mathematics and Computation $191(2), 334-346$.

[10] Golbabai, A., Kermati, B., 2008. Modified homotopy perturbation method for solving Fredholm integral equations. Choas, Solitions and Fractals 37 (5), 1528-1537.

[11] He, J.H., 1999. Homotopy perturbation technique Computer methods in Applied Mechanics and Engineering, 178, 257-262.

[12] He, J.H., 2000. A coupling method of homotopy perturbation technique for nonlinear problems. International Journal of Non-linear Mechanics 35, (1), 37-43.

[13] He, J.H., 2003. Homotopy perturbation method: a new nonlinear analytical technique. Applied Mathematics and Computation 135, 73-79.

[14] He, J.H., 2004. Comparison homotopy perturbation method and homotopy analysis method. Applied Mathematics and Computation 156, 527-539.

[15] He, J.H., 2004. The homotopy perturbation method for nonlinear oscillators with discontinuities. Applied Mathematics and Computation 151, 287-292.

[16] He, J.H., 2005. Limit cycle and bifurcation of nonlinear problems. Chaos, Solitons and Fractals 26 (3), 827-833. 
[17] He, J.H., 2005. Application of homotopy perturbation method to nonlinear wave equations. Chaos, Solitons and Fractals 26, 695-700.

[18] He, J.H., 2006. Homotopy perturbation method for solving boundary value problems. Physics Letters A 350, 87-88.

[19] Mohyud-Din, S.T., Yildirim, A., Demirli, G., 2010. Traveling wave solutions of Whitham-Broor-Kaup equations by homotopy perturbation method. Journal of King Saud University-Science 22(3), 173-176.

[20] Odibat, Z., Momani, S., 2008. Modified homotopy perturbation method: application to quadratic Riccati differential equation of fractional order. Chaos, Solitions and Fractals 36 (1), 167-174.

[21] Raftari, B., Yildirim, A., 2010. The application of homotopy perturbation method for MHD flows of UCM fluids above porous stretching sheets. Computers \& Mathematics with Applications 59 (10), 3328-3337.

[22] Shakeri, Fatemeh, Dehghan, Mehdi, 2008. Solution of delay differential equations via a homotopy perturbation method, Mathematical and Computer Modeling 48, 486-498.

[23] Siddiqui, A.M., Mahmood, R., Ghori, Q.K., 2008. Homotopy perturbation method for thin flow of a third grade fluid down an inclined plane. Chaos, Solitions and Fractals 35, 140-147.
[24] Sun, F.Z., Gao, M., Lei, S.H., et al., 2007. The fractal dimension of the fractal model of drop-wise condensation and its experimental study. International Journal of Nonlinear Sciences and Numerical Simulation 8 (2), 211-222.

[25] Wang, H., Fu, H.M., Zhang, H.F., et al., 2007. A practical thermo-dynamic method to calculate the best glass-forming composition for bulk metallic glasses. International Journal of Nonlinear Sciences and Numerical Simulation 8 (2), 171-178.

[26] Xu, L., H, J.H., Liu, Y., 2007. Electrospun nano-porous spheres with Chines drug. International Journal of Nonlinear Sciences and Numerical Simulation 8 (2), 199-202.

[27] Yildirim, A., Gulkanat, Y., 2010. Analytical approach to fractional Zakharov-Kuznetsov equations by He's homotopy perturbation method. Communications in Theoretical Physics 53(6), 1005-1010.

[28] Yildirim, A., Mohyud-Din, S.T., Zhang, D.H., 2010. Analytical solutions to the pulsed Klein-Gordon equation using Modified Variational Iteration Method (MVIM) and Boubaker Polynomials Expansion Scheme (BPES). Computers \& Mathematics with Applications 59 (8), 2473-2477. 